\title{
Comparisons of neutrophil, monocyte, eosinophil, basophil and lymphocyte ratios among the fibromyalgia syndrome and healthy
} individuals

\author{
Nihal Tezel $^{1 *}$, Selcan Gültuna ${ }^{2}$
}

\begin{abstract}
Objective: To evaluate the basophil lymphocyte ratio (BLR) and eosinophil lymphocyte ratio (ELR) values in the fibromyalgia syndrome (FMS) that previously reported being used as an indicator of inflammation in autoimmune rheumatic diseases and smokers.

Material and Methods: We retrospectively analyzed 4500 fibromyalgia (FM) patients who were registered in the network system with the M79-ICD code and 2000 healthy controls. A total of 216 FM patients and 194 healthy controls were included in the study.

Results: The blood BLR levels were significantly higher in FM patients than in healthy controls. $(\mathrm{p}<0,02)$. The two groups did not show significant differences in terms of the other parameters $(p>0.05)$.

Conclusion: In the present study, neutrophil lymphocyte ratio (NLR), monocyte lymphocyte ratio (MLR), platelet lymphocyte ratio (PLR), ELR, platelet distribution width (PDW) are independent markers for early diagnosis and for the inflammatory predictive process. BLO levels were low revealed. To use these rates as disease markers should be supported by large-scale studies.
\end{abstract}

Keywords: Neutrophil-lymphocyte ratio; monocyte-lymphocyte ratio; eosinophil-lymphocyte ratio; basophillymphocyte ratio; fibromyalgia syndrome; inflammatory marker.

\section{Introduction}

Fibromyalgia syndrome (FMS) is a common chronic pain syndrome with female predominance characterized by diffuse stiffness, pain, tenderness, and somatic symptoms (like sleep problems, headache, fatigue) (1). The incidence of FMS in the general population is $2-4 \%$ (2). Although the etiology and pathogenesis of FMS have remained unknown until now, genetic and epigenetic causes have been suggested in which the FMS etiopathogenesis pain regulation system is impaired (3). In FMS, which cannot be diagnosed by any laboratory or imaging, the diagnosis can be made with clinical and examination findings. In FMS, where somatic symptoms are intensely observed, it is important to exclude other diseases in which these symptoms can be common. Therefore, laboratory support is very important in the diagnosis. According to previous findings, an increase of Pro-inflammatory cytokines like IL 8, IL 6 has been detected in fibromyalgia (FM) patients (4).
However, these biomarkers cannot be used in clinical practice. In recent years, neutrophil- neutrophil-lymphocyte (NLR) and Platelet Distribution Width (PDW) have been identified as two important systemic inflammation markers.

And, many studies have reported that NLR and PDW were associated with inflammatory activity and prognosis in FMS (5). However, there are studies indicating otherwise (6).

The aim of the study is to evaluate the BLR and ELR values in FMS that previously reported to be used as an indicator of inflammation in autoimmune rheumatic diseases and smokers $(7,8)$. 


\section{Material and Methods}

Patients diagnosed with fibromyalgia (FM) who applied to the physical therapy and rehabilitation outpatient clinic between January 2018 and November 2018 were scanned. Patients with disease that may affect the blood count (diabetes, B12, vitamin D, ferritin deficiency, hypothyroidism, hypercholesterolemia, high acute phase reactants or have comorbidities ) both patient and control groups were excluded from the study.

We retrospectively analyzed $4500 \mathrm{FM}$ patients who were registered in the network system with the M79-ICD code and 2000 healthy controls. 4284 FM patients and the 1806 healthy controls excluded from the study due to comorbidities and the reasons that can be affected the blood values. A total of 216 FM patients and 194 healthy controls were included in the study. All participants' age, gender, lökosit, neutrophil, lymphocyte, eosinophil, basophil, monocyte and platelet counts; PDW; and MPV data were recorded. The study protocol was approved by the local Ethics Committee. (approve date:04.02.2019, approve number:59/06) The study was conducted in accordance with the principles of the Declaration of Helsinki.

\section{Statistical analysis}

The statistical analysis was performed using the SPSS for Windows 11.5 package program (SPSS Inc., Chicago, IL, USA). The variables were investigated using visual (histograms, probability plots) and analytical methods (Kolmogorov-Smirnov / Shapiro-Wilk's test) to determine whether or not normally distributed.
Normally distributed continuous variables were expressed as mean \pm standard deviation (s.d) while the continuous variables that do not have normal distribution were expressed as median (minimum-maximum). Also, categorical variables were summarized as counts (percentages). Comparisons of normally distributed continuous variables between groups were tested using the Student's test. For non-normally continuous variables, differences between groups were tested using the MannWhiney $\mathrm{U}$ test. ROC curve analysis was used to test the hallmark of BLO in predicting FM. A two-sided p-value of less than 0.05 was considered as statistically significant.

\section{Results}

Age and gender data for 174 FM patients and 194 healthy controls were shown in Table 1. A total of 196 FM patients ( 176 women, 20 men) and 194 healthy control (174 women, 20 men) included in the study. The mean age for the patients and control groups were 44,43 $\pm 8,69$ and $42,53 \pm 9,60$ years respectively. There were no statistically significant age and gender differences between the groups.

Laboratory data has been shown in Table 2. The blood BLR levels were significantly higher in FM patients than in healthy controls. $(\mathrm{p}<0,02)$

The two groups did not show significant differences in terms of the other parameters $(\mathrm{p}>0.05)$.

ROC analysis The area under the curve (AUC) was 0.42 .

Table 1 Demographics of the patients (SD: Standard Deviation)

\begin{tabular}{|lcc|}
\hline Male (\%) & Group 1 $(\mathbf{n = 2 1 6})$ & Group 2 $(\mathbf{n = 1 9 4})$ \\
Female (\%) & $20(9,3)$ & $20(10,3)$ \\
Age, Mean+SD & $196(90,7)$ & $174(89,7)$ \\
\hline
\end{tabular}

Table 2 Comparison of NLR, PLR, ELR, BLR, MLO, MPV, RDW between fibromyalgia patients and healthy controls

\begin{tabular}{|c|c|c|c|}
\hline & $\begin{array}{c}\text { Group 1 } \\
(n=216)\end{array}$ & Group $2(n=194)$ & $\mathbf{p}^{*}$ \\
\hline NLR, median min-max & $1,84(0,54-7,00)$ & $1,80(0,29-11,33)$ & 0,262 \\
\hline PLR, median min-max & $119,41(41,67-823,33)$ & $119,87(54,55-783,33)$ & 0,702 \\
\hline ELR, median min-max & $0,58(0-0,67)$ & $0,06(0-0,36)$ & 0,084 \\
\hline BLR, median min-max & $0(0-1,11)$ & $0(0-1,4)$ & $0,002 *$ \\
\hline MLO, median min-max & $0,21(0,06-6,33)$ & $0,21(0,03-1,33)$ & 0,678 \\
\hline MPV, mean $\pm S D$ & $8,75 \pm 1,32$ & $8,94 \pm 1,39$ & 0,370 \\
\hline RDW, mean \pm SD & $13,76 \pm 1,31$ & $13,67 \pm 1,22$ & 0,434 \\
\hline WBC, mean \pm SD & $7,41 \pm 1,73$ & $7,25 \pm 1,50$ & 0,489 \\
\hline NEU, mean \pm SD & $4,38 \pm 1,422$ & $4,18 \pm 1,22$ & 0,264 \\
\hline MONO, mean \pm SD & $0,51 \pm 0,18$ & $0,49 \pm 0,14$ & 0,337 \\
\hline LYM, mean \pm SD & $2,30 \pm 0,59$ & $2,29 \pm 0,61$ & 0,607 \\
\hline EOS, mean \pm SD & $0,16 \pm 0,13$ & $0,17 \pm 0,10$ & 0,113 \\
\hline BASO, mean \pm SD & $0,04 \pm 0,15$ & $0,05 \pm 0,15$ & 0,463 \\
\hline PLT, mean \pm SD & $269,65 \pm 58,34$ & $272,30 \pm 58,93$ & 0,596 \\
\hline PDW, mean \pm SD & $16,37 \pm 1,32$ & $16,16 \pm 1,22$ & 0,176 \\
\hline
\end{tabular}

NLR: neutrophil lymphocyte ratio; PLR: platelet lymphocyte ratio; ELR: eosinophil lymphocyte ratio; BLR:basophil lymphocyte ratio; MLO:monocyte lymphocyte ratio; MPV:mean platelet volume; RDW: Red Blood cell distribution width; WBC: white Blood cell; NEU: neutrophil; MONO:monocyte; LYM: lymphocyte; EOS: eosinophil; BASO: basophil; PLT: platelet; PDW: platelet distribution width; min-max:minimummaximum; SD: Standard deviation. 


\section{Discussion}

FMS is a multifactorial disease with unknown etiology. In early diagnosis and treatment, typically there are no laboratory abnormalities specifically associated with FMS. In FM patients, NLR, PLR rates, MPV and RDW Blood distribution parameters have been previously evaluated in various studies, and different results have been revealed (5). To our knowledge, this is the first study to evaluate NLR, MLR, ELR, and BLR levels in FMS. In the present study, we did not find any difference in NLR, PLR, ELR ratios and MPV and RDW values between FM patients and healthy controls. In FM patients, BLR was lower than the control group.

Zhang et al (7) found that NLR, MLR levels were significantly higher in the inflammatory rheumatic disease and were closely related to AFR. Similarly, Uslu et al (9) found the relationship between NLR and PLR levels and DAS-28 scores. And they indicated that these ratios can be used as inflammatory markers in rheumatoid arthritis.

Aktürk et al. (5) found NLR levels high in FM patients but did not find a correlation with AFR. The NLR has been reported as a prognostic marker to determine the systemic inflammatory response.

Similar to our study, Karataş et al. (6) did not found any difference between healthy controls and FM patients in NLR, MLR, and PLR levels. They suggested that FMS is not an inflammatory disease.

Taşoğlu et al. (10) evaluated the correlation between severe knee osteoarthritis with NLR and reported that patients with severe knee osteoarthritis had higher BLR and NLR values compared to those with mild knee osteoarthritis. However, in this study, there was a significant difference in ages between the groups. Fest et al. (11) found the distribution of the NLR and PLR was different between age categories. Also in osteoarthritis (OA), the inflammatory reaction can trigger the OA changes (12). These differences can be explained by age or inflammation in severe osteoarthritis.

Ilgun et al. did not find an association between FM NLR and found a correlation between tender scores with PLR scores (13). In this study, the diagnosis of FM was made according to the 1990 ACR criteria. However, clinical experience and epidemiological data show that FMS patients frequently report other symptoms such as sleep disturbances, fatigue, irritable bowel syndrome, and others.

Taş et al. (14) investigated the NLR and PLR in rest leg syndrome, one of the central sensitization syndromes such as FMS. They found no difference similar to our study.

Qin et al (15) investigated NLR and PLR in patients with SLE patients. The rates were significantly higher in the patient group and correlated with $\mathrm{C}$-Reactive protein and nephritis.

Monocyte-lymphocyte ratio (MLR), were not evaluated in FMS before. It has been related to diabetic retinopathy and the predictive value of the prognosis of some tumors $(16,17)$. In the present study, we did not find any MLO differences between groups.
Eosinophil and basophil in normal blood tend to be low as has been known, increases in these two types of leukocytes are used to reflect allergic diseases and parasitic infections. In our study, we found that BLR levels were decreased. Like the present study, a clinical study also found a decrease in basophil ratio in SLE patients. In this study, although BLR levels were found to be low in SLE patients, they did not find any correlation with inflammatory and immune markers. Indeed, they indicate that basophil cells are effective in SLE pathogenesis, there is no such literature on FM (7).

Hematologic parameters such as neutrophil, lymphocyte, and platelet counts can easily be affected by various conditions such as ethnicity, age, sex, eating habits, and environmental factors (18). One of the reasons for differences in results between similar studies can be explained by this.

The role of systemic inflammation in the pathogenesis of FMS has not been clear. We can see significant differences in blood distribution parameters are mostly detected in inflammatory processes. The meaning of inflammatory mechanisms and blood distribution parameters in FMS should be supported by much larger studies.

The strengths of our study are that the number of patients screened was high, blood counts ratios used as an inflammatory marker in some diseases which have not been previously evaluated in FMS were investigated, and we have determined methodological exclusion criteria well.

Limitation of the study as the network database is examined. The patients cannot be evaluated in terms of disease severity or quality of life.

\section{Conclusion}

Discovering new biomarkers of inflammation becomes important in order to help diagnostic accuracy and provide prognostic information about fibromyalgia. Our study demonstrated that BLR levels are markedly decreased in FMS. The literature has comprehensive results about the fibromyalgia and NLR, MLR, PLR, ELR, PDW relations.

In the present study, NLR, MLR, PLR, ELR, PDW are independent markers for early diagnosis and for the inflammatory predictive process. BLO levels were low revealed. It is our opinion that to use these rates as disease markers must be supported by large-scale studies.

\section{Acknowledgments: None}

Author Contributions: NT, SG: Design of research, data collection, SG; Statistical analysis, NT: Writing of manuscript and revisions.

Conflict of Interest: The authors declare that they have no conflict of interest

\section{References}

1. Lichtenstein A, Tiosano S, Amital H. The complexities of fibromyalgia and its comorbidities . Curr Opin Rheumatol. 2017, 30:94-100. 
2. Clauw DJ, D'Arcy Y, Gebke K, Semel D, Pauer L, Jones KD. Normalizing fibromyalgia as a chronic illness. Postgrad Med 2018; 130: 9-18.

3. Cabo-Meseguer A, Cerdá-Olmedo G, Trillo-Mata JL. Fibromyalgia: Prevalence, epidemiologic profiles and economic costs. Med Clin 2017;149:441-8.

4. Rodriguez-Pinto I, Agmon-Levin N, Howard A, Shoenfeld Y. Fibromyalgia and cytokines. Immunol Lett. 2014;161(2):200-203.

5. Aktürk S, Büyükavcı R. Evaluation of BLRod neutrophillymphocyte ratio and platelet distribution width as inflammatory markers in patients with fibromyalgia. Clin Rheumatol. 2017;36(8):1885-1889.

6. Karataş G, Gündüz R. The Significance of inflammation markers in complete blood count in patients with fibromyalgia. Med Sci Discov. $2020 ; 7(1): 364-7$.

7. Zhang Y, Yin Y, Kuai S, Shan Z, Pei H, Wang J. Combination of neutrophil to lymphocyte ratio and platelet to lymphocyte ratio as diagnostic biomarker for rheumatoid arthritis. Int J Clin Exp Med. 2016; 9: 22076- 22081 .

8. Çekici Y, Yılmaz M, Seçen O. New inflammatory indicators: association of high eosinophil to-lymphocyte ratio and low lymphocyte-to-monocyte ratio with smoking. Journal of International Medical Research.2019;(47)9:4292-4303.

9. Uslu AU, Kucuk A, Sahin A, Ugan Y, Y1lmaz R, Güngör T et al. Two new inflammatory markers associated with disease activity score-28 in patients with rheumatoid arthritis: Neutrophillymphocyte ratio and platelet-lymphocyte ratio. Int $\mathrm{J}$ Rheum Dis 2015; 18( 7): 731-735.

10. Tasoglu O, Boluk H, Onat SS, Tasoglu I, Ozgirgin N. Is blood neutrophil-lymphocyte ratio an independent predictor of knee osteoarthritis severity?Clin Rheumatol 2016;35 (6):1579-1583.
11. Fest J, Ruiter R, Ikram MA, Voortman T, Van Eijck CHJ, Stricker BH. Reference values for white blood-cell-based inflammatory markers in the Rotterdam Study: a population-based prospective cohort study.Sci Rep 2018; 8:10566.

12. Mora JC, Przkora R, Cruz-Almeida Y. Knee osteoarthritis: pathophysiology and current treatment modalities. J Pain Res. 2018;11:2189-96.

13. İlgün E, Akyürek Ö, Kalkan AO, Demir F, Demirayak M, Bilgi M Neutrophil/Lymphocyte Ratio and Platelet/Lymphocyte Ratio in Fibromyalgia. Eur J Gen Med. 2016;13(2):100-4.

14. Tak AZA, Şengül Y. Evaluation of Inflammation with Neutrophil-tolymphocyte Ratio and Platelet-to-lymphocyte Ratio in Restless Legs Syndrome. Turk J Neurol. 2018; 24(3): 259-263.

15. Qin B, Ma N, Tang Q, Wei T, Yang M, Fu H, et al. Neutrophil to lymphocyte ratio (NLR) and platelet to lymphocyte ratio (PLR) were useful markers in assessment of inflammatory response and disease activity in SLE patients. Mod Rheumatol.2016; 26(3), 372-6.

16. Feng F, Tian Y, Liu S, Zheng G, Liu Z, Xu G, et al. Combination of PLR, MLR, MWR, and tumor size could significantly increase the prognostic value for gastrointestinal stromal tumors. Medicine 2016;95:3248.

17. Li J, Jiang R, Liu WS, Liu Q, Xu M, Feng QS, et al. A large cohort study reveals the association of elevated peripheral BLRod lymphocyte-to-monocyte ratio with favorable prognosis in nasopharyngeal carcinoma. PLoS ONE 2013;8:83069.

18. El-Hazmi MA, Warsy AS. Normal reference values for hematological parameters, red cell indices, HB A2 and HB F from early childhood through adolescence in Saudis. Ann Saudi Med 2001;21:165-169.

Copyright (C) 2020 The Author(s); This is an open-access article distributed under the terms of the Creative Commons Attribution License (http://creativecommons.org/licenses/by/4.0), (CC BY NC) which permits unrestricted use, distribution, and reproduction in any medium, provided the original work is properly cited. International journal of Medical Science and Discovery. 